\section{Standardized monitoring of cytomegalovirus- specific immunity can improve risk stratifica- tion of recurrent cytomegalovirus reactivation after hematopoietic stem cell transplantation}

\author{
Eva Wagner-Drouet,,$^{1, *}$ Daniel Teschner $r^{1, *}$ Christine Wolschke, ${ }^{2}$ Dietlinde \\ Janson, ${ }^{2}$ Kerstin Schäfer-Eckart, ${ }^{3}$ Johannes Gärtner, ${ }^{3}$ Stephan Mielke, ${ }^{4,5}$ Martin \\ Schreder, ${ }^{4,8}$ Guido Kobbe, ${ }^{6}$ Mustafa Kondakci, ${ }^{6}$ Inken Hilgendorf, ${ }^{7}$ Marie von \\ Lilienfeld-Toal, ${ }^{7}$ Stefan Klein, ${ }^{8}$ Daniela Heidenreich, ${ }^{8}$ Sebastian Kreil, ${ }^{8}$ Mareike \\ Verbeek, ${ }^{9}$ Sandra Graß, ${ }^{9}$ Markus Ditschkowski, ${ }^{10}$ Tanja Gromke, ${ }^{10}$ Martina \\ Koch, ${ }^{11,+}$ Monika Lindemann, ${ }^{12}$ Thomas Hünig, ${ }^{13}$ Traudel Schmidt, ${ }^{14}$ Anne \\ Rascle,${ }^{14}$ Harald Guldan,,${ }^{14}$ Sascha Barabas,${ }^{14}$ Ludwig Deml, ${ }^{14}$ Ralf Wagner ${ }^{14,15}$ \\ and Daniel Wolff ${ }^{16}$
}

\begin{abstract}
${ }^{1}$ Department of Hematology, Medical Oncology, and Pneumology, University Medical Center of the Johannes Gutenberg University, Mainz, Germany; '2Department of Stem Cell Transplantation, University Medical Center Hamburg-Eppendorf, Germany; ${ }^{3}$ Oncology, Hematology and Bone Marrow Transplantation Unit, Klinikum Nord, Nürnberg, Germany; ' Department of Medicine II, University Medical Center, Würzburg, Germany; ${ }^{5}$ Department of Laboratory Medicine, CAST, Karolinska Institutet and University Hospital, Stockholm, Sweden; ${ }^{6}$ Department of Hematology, University Hospital Düsseldorf, Medical Faculty, Heinrich Heine University, Düsseldorf, Germany; ${ }^{7}$ Klinik für Innere Medizin II, Abteilung für Hämatologie und Internistische Onkologie,

Universitätsklinikum, Jena, Germany; ${ }^{8}$ Department of Hematology and Oncology, UMM University Medical Center Mannheim, University of Heidelberg, Mannheim, Germany; III Medical Department, Hematology and Oncology, Klinikum Rechts der Isar, Technical University, Munich, Germany; ${ }^{10}$ Innere Klinik, Tumorforschung, University Hospital Essen, Germany; ${ }^{11}$ Department of Hepatobiliary Surgery and Transplantation, University Medical Center Hamburg-Eppendorf, Germany; ${ }^{12}$ Institute for Transfusion Medicine, University Hospital, Essen, Germany; ${ }^{13}$ Institute of Virology and Immunobiology, University Medical Center, Würzburg, Germany; ${ }^{14}$ Lophius Biosciences, Regensburg, Germany; ${ }^{15}$ Institute of Clinical Microbiology and Hygiene, University Medical Center Regensburg, Germany and ${ }^{16}$ Department of Internal Medicine III, Hematology and Oncology, University Medical Center Regensburg, Germany.
\end{abstract}

*EW-D and DT contributed equally as co-first authors.

${ }^{s}$ Current address: First Department of Medicine, Center for Oncology and Hematology, Wilhelminenspital, Vienna, Austria

${ }^{\ddagger}$ Current address: Department of General, Visceral and Transplantation Surgery, University Medical Center of the Johannes Gutenberg University, Mainz, Germany

\section{ABSTRACT}

$\mathrm{R}$ ecurrence of cytomegalovirus reactivation remains a major cause of morbidity and mortality following allogeneic hematopoietic stem cell transplantation. Monitoring cytomegalovirus-specific cellular immunity using a standardized assay might improve the risk stratification of patients. A prospective multicenter study was conducted in 175 intermediate- and high-risk allogeneic hematopoietic stem cell transplant recipients under preemptive antiviral therapy. Cytomegalovirusspecific cellular immunity was measured using a standardized interferon$\gamma$ enzyme-linked immunospot assay (T-Track ${ }^{\circledR} \mathrm{CMV}$ ). The primary aim was to evaluate the suitability of measuring cytomegalovirus-specific immunity after the end of treatment for a first cytomegalovirus reactivation to predict recurrent reactivation. Forty of 101 (39.6\%) patients with a first cytomegalovirus reactivation experienced recurrent reactivations, mainly in the high-risk group (cytomegalovirus-seronegative donor/cytomegalovirus-seropositive recipient). The positive predictive value of $\mathrm{T}$-Track ${ }^{\circledR} \mathrm{CMV}$ (patients with a negative test after the first reactivation who experienced at least one recurrent reactivation) was $84.2 \%$ in high-risk patients. Kaplan-Meier analysis revealed a higher probability of recurrent cytomegalovirus reactivation in high-risk patients with a
Haematologica 2021

Volume 106(2):363-374

\section{Correspondence:}

DANIEL WOLFF

daniel.wolff@ukr.de

RALF WAGNER

ralf.wagner@ukr.de

Received: June 10, 2019.

Accepted: December 18, 2019.

Pre-published: December 26, 2019.

https://doi.org/10.3324/haematol.2019.229252

(C)2021 Ferrata Storti Foundation

Material published in Haematologica is covered by copyright. All rights are reserved to the Ferrata Storti Foundation. Use of published material is allowed under the following terms and conditions:

https://creativecommons.org/licenses/by-nc/4.0/legalcode. Copies of published material are allowed for personal or internal use. Sharing published material for non-commercial purposes is subject to the following conditions:

https://creativecommons.org/licenses/by-nc/4.0/legalcode, sect. 3. Reproducing and sharing published material for commercial purposes is not allowed without permission in writing from the publisher. 
negative test after the first reactivation (hazard ratio $2.73 ; P=0.007$ ). Interestingly, a post-hoc analysis considering T-Track ${ }^{\circledR}$ CMV measurements at day 100 after transplantation, a time point highly relevant for outpatient care, showed a positive predictive value of $90.0 \%$ in high-risk patients. Our results indicate that standardized cytomegalovirus-specific cellular immunity monitoring may allow improved risk stratification and management of recurrent cytomegalovirus reactivation after hematopoietic stem cell transplantation. This study was registered at www.clinicaltrials.gov as \#NCT02156479.

\section{Introduction}

Cytomegalovirus (CMV) infection and disease remain a serious cause of morbidity and mortality after allogeneic hematopoietic stem cell transplantation (HSCT). ${ }^{1-3}$ Thanks to efficient diagnosis and management of CMV, the incidence of death from CMV disease after HSCT has dropped to $<10 \%{ }^{49}$ Adequate risk stratification is essential to identify and properly manage patients at risk of CMV reactivation. The main risk factors include donor (D) and recipient (R) CMV serostatus ( $\mathrm{D}-/ \mathrm{R}+$ defining high-risk patients), the use of mismatched or unrelated donors, graft-versus-host disease (GvHD), and intense immunosuppression. ${ }^{1,2}$ Close monitoring during the first 100 days after transplantation, in accordance with current guidelines, has greatly reduced the incidence of CMV-related complications. However, recurrent and late-occurring CMV reactivation remain major lifethreatening issues, and effective strategies for the prevention of late CMV disease prevail as an unmet medical need. This is particularly critical in outpatient care more than 100 days after HSCT when patients are less frequently monitored. Studies clearly identified a delay in global and CMVspecific immune reconstitution as a major risk factor for recurrent and late-onset CMV reactivation. ${ }^{10-13}$ Several $\mathrm{CMV}$-specific immune monitoring assays have been described. They are based on the quantification of the number and/or functionality of immune cells targeted against CMV, using flow cytometry detection, an enzyme-linked immunosorbent assay (ELISA) or enzyme-linked immunospot (ELISpot). ${ }^{14,15}$ Multiple studies demonstrated the suitability of these methods for predicting recurrent and/or late CMV reactivation, resulting in the emergence of new risk stratification models based on the monitoring of CMV-specific cell-mediated immunity (CMV-CMI) together with CMV viral load. ${ }^{8,16-25}$ The lack of standardized assays does, however, render the comparison of most reported results difficult. Two standardized CMV-specific interferon (IFN)- $\gamma$ ELISpot assays, based on the in vitro stimulation of peripheral blood mononuclear cells (PBMC) with IE-1 and pp65 peptides (T-SPOT ${ }^{\circledR}$.CMV) or proteins (T-Track ${ }^{\circledR}$ $\mathrm{CMV}$ ), have been described. T-Track ${ }^{\circledR} \mathrm{CMV}$ is highly sensitive due to the use of urea-formulated T-activated ${ }^{\circledR}$ IE- 1 and pp65 proteins, resulting in the activation of a broad spectrum of CMV-specific effector cells (including $\mathrm{CD} 4^{+}, \mathrm{CD} 8^{+}$ and NK cells). ${ }^{14,26-28}$ One study reported the utility of T$\mathrm{SPOT}^{\circledast}$.CMV to predict the risk of a first treatment-requiring CMV reactivation after $\mathrm{HSCT} .{ }^{29}$ Here we describe - to the best of our knowledge for the first time - the utility of the $\mathrm{T}$-Track ${ }^{\oplus} \mathrm{CMV}$ assay to predict recurrent and late-onset CMV reactivation after HSCT.

\section{Methods}

\section{Study design and participants}

A prospective multicenter study was conducted in 175 interme- diate- and high-risk ( $\mathrm{D}+/ \mathrm{R}+, \mathrm{D}+/ \mathrm{R}-, \mathrm{D}-/ \mathrm{R}+)$ allogeneic HSCT recipients. Recipients of a first-time bone marrow or peripheral blood transplantation from either a matched sibling, matched unrelated or mismatched unrelated donor of any gender and race, aged at least 18 years, and scheduled for preemptive antiviral therapy were eligible for participation in this study, which was approved by the relevant ethics committees (DIMDI's registration number 00008544; University of Regensburg's approval number 13-122-0282). Additional information is provided in the Online Supplementary Methods.

\section{Viral load measurement}

CMV load was measured by quantitative polymerase chain reaction using validated, non-standardized protocols and equipment (Online Supplementary Methods). CMV reactivation was defined as a CMV viral load requiring antiviral treatment based on center-specific guidelines and/or physician's decision. Accordingly, the term "CMV reactivation" will thereafter refer to antivirallytreated $\mathrm{CMV}$ reactivation.

\section{Measurement and analysis of CMV-specific cell-mediated immunity}

Blood collection, peripheral blood mononuclear cell isolation and T-Track ${ }^{\circledR} \mathrm{CMV}$ assays (Lophius Biosciences $\mathrm{GmbH}$, Regensburg, Germany) were performed as described ${ }^{26,27,30}$ and as detailed in the Online Supplementary Methods. IFN- $\gamma$ ELISpot test results were interpreted on the basis of square-root-transformed spot-forming cells (sqrt-SFC). Briefly, a test was considered positive if the mean of four replicate sqrt-SFC for 200,000 cells (SRM) resulting from IE-1 and/or pp65 stimulation was $\geq \operatorname{sqrt}(10)$ and if the difference of the mean of sqrt-SFC of the stimulated condition to that of the unstimulated condition (SRM[stimulated] SRM[unstimulated]) was $\geq 0.742$.

\section{Lymphocyte subpopulation count determination}

Lymphocyte subpopulations were characterized by flow cytometry from the same peripheral blood mononuclear cells and absolute cell counts were calculated using the peripheral blood absolute lymphocyte count determined at the same visit (Online Supplementary Methods).

\section{Statistical analysis}

Calculations were performed with SAS 9.4 software, as detailed in the Online Supplementary Methods. Statistical analyses are presented for high-risk (D-/R+) and all patients. Differences in IE-1and pp65-specific SFC distributions between groups were tested using the Mann-Whitney $U$ test. Qualitative (positive/negative) test results were compared using a $\chi^{2}$ test. The probability of recurrent CMV reactivation according to qualitative test results was estimated using Kaplan-Meier curves. Hazard ratio (HR) estimates were obtained by Cox regression analysis and differences in CMV reactivation probability between groups were tested using a log-rank test. Receiver operating characteristic (ROC) curves were generated and area under the curve (AUC) estimates were obtained by logistic regression. Two-sided $P$-values $<0.05$ were considered statistically significant. 


\section{Results}

\section{Patients' characteristics}

One hundred and seventy-five allogeneic HSCT recipients were enrolled. Twenty-one patients were excluded from the analysis, due to either protocol violation $(n=8)$ or the absence of valid T-Track ${ }^{\circledast}$ CMV test results $(n=13)$. The study flow diagram is shown in Online Supplementary Figure S2. Of the 154 HSCT recipients included in the final analysis, 101 (65.5\%) experienced at least one treatmentrequiring CMV reactivation (hereafter designated as "CMV reactivation") up to day 225 after transplantation (Table 1). Eight patients (4.6\%) were diagnosed with CMV disease and $69(44.8 \%)$ with GvHD (Table 1 and Online Supplementary Table S2). The majority of patients with GvHD (50/69 [72.5\%] of all patients and 33/40 [82.5\%] of $\mathrm{D}-/ \mathrm{R}+$ patients) experienced at least one episode of CMV reactivation. This is in line with the strong immunosuppressive effect of steroids used for the treatment of GvHD. ${ }^{31,32}$ Of the 101 patients who had a CMV reactivation, $65(64.4 \%)$ belonged to the $\mathrm{D}-/ \mathrm{R}+$ high-risk group (Table 2). Sixty-one (60.4\%) patients experienced only one CMV reactivation while 40 patients developed either two $(n=24)$ or three $(n=16)$ CMV reacti-

Table 1. Patients' characteristics.

\begin{tabular}{|c|c|}
\hline Study population, N (\%) & $154(100 \%)$ \\
\hline $\begin{array}{l}\text { Gender, n (\%) } \\
\text { Male } \\
\text { Female }\end{array}$ & $\begin{array}{l}88(57.1) \\
66(42.9)\end{array}$ \\
\hline Age in years, median (range) & $58(20-75)$ \\
\hline $\begin{array}{l}\text { Underlying disease, n (\%) } \\
\text { Acute myeloid leukemia } \\
\text { Myelodysplastic syndrome } \\
\text { Acute lymphoid leukemia } \\
\text { Non-Hodgkin lymphoma } \\
\text { Multiple myeloma } \\
\text { Osteomyelofibrosis } \\
\text { Chronic myeloid leukemia } \\
\text { Chronic lymphoid leukemia } \\
\text { Severe aplastic anemia }\end{array}$ & $\begin{array}{l}79(51.3) \\
24(15.6) \\
17(11.0) \\
12(7.8) \\
10(6.5) \\
4(2.6) \\
3(1.95) \\
3(1.95) \\
2(1.3)\end{array}$ \\
\hline $\begin{array}{l}\text { Donor (D) / recipient (R) CMV serostatus, n (\%) } \\
\text { D+/R+ } \\
\text { D+/R- } \\
\text { D-/R+ }\end{array}$ & $\begin{array}{l}53(34.4) \\
19(12.3) \\
82(53.3)\end{array}$ \\
\hline $\begin{array}{l}\text { Stem cell source, n (\%) } \\
\text { Bone marrow } \\
\text { Peripheral blood }\end{array}$ & $\begin{array}{c}9(5.8) \\
145(94.2)\end{array}$ \\
\hline $\begin{array}{l}\text { Donor source, } \mathrm{n}(\%) \\
\text { Matched sibling } \\
\text { Matched unrelated donor } \\
\text { Mismatched unrelated donor }\end{array}$ & $\begin{array}{l}31(20.1) \\
92(59.8) \\
31(20.1)\end{array}$ \\
\hline $\begin{array}{l}\text { Conditioning regimen, } \mathrm{n}(\%) \\
\text { Non-myeloablative } \\
\text { Myeloablative, standard } \\
\text { Myeloablative, toxicity-reduced }\end{array}$ & $\begin{array}{l}41(26.6) \\
75(48.7) \\
38(24.7)\end{array}$ \\
\hline At least one treatment-requiring CMV reactivation, $\mathrm{n}(\%)$ & $101(65.6)$ \\
\hline CMV disease, $\mathrm{n}(\%)$ & $8(4.6)$ \\
\hline Graft-versus-host disease, n (\%) & $69(44.8)$ \\
\hline Infections other than CMV (after day 45), n (\%) & $52(33.8)$ \\
\hline Death, $\mathrm{n}(\%)$ & $21(13.6)$ \\
\hline
\end{tabular}

CMV: cytomegalovirus. vations (hereafter referred to as "recurrent CMV reactivation") (Table 2). Most of the HSCT recipients who had recurrent CMV reactivation were high-risk patients (37/40 [92.5\%]) (Table 2). Therefore, we focused on highrisk patients as the clinically relevant population with regards to the risk of CMV recurrence after HSCT. The median (range) time to CMV reactivation in $\mathrm{D}-/ \mathrm{R}+$ patients for the first, second and third CMV reactivation was 37 (19-58), 109 (63-188) and 174 (119-219) days after transplantation, respectively (Online Supplementary Figure $S 3 B$ ). The respective median (range) CMV viral load is presented in Figure 1.

\section{Measurement of CMV-specific cell-mediated immunity over time after hematopoietic stem cell \\ transplantation}

CMV-CMI was evaluated using a standardized IFN- $\gamma$ ELISpot-based assay (T-Track ${ }^{\circledast}$ CMV) ${ }^{26,27,30}$ A total of 647 valid test results were included in the analysis. The distribution of spot-forming cells (SFC) was analyzed over time after transplantation in response to the CMV proteins IE1 and pp65 (Online Supplementary Figure S4A). Overall, the response to IE-1 antigen was lower than that to pp65 throughout the study, especially in D-/R+ patients. A significant increase in the response to pp65 was apparent in $\mathrm{D}-/ \mathrm{R}+$ patients over time (Online Supplementary Figure S4A). Accordingly, while the proportion of IE-1-positive tests remained low (up to $33.3 \%$ around day 145 ), that of pp65-positive tests increased to $64.5 \%$ in $\mathrm{D}$-/R+ patients (Online Supplementary Figure S4B). Interestingly, the percentage of T-Track ${ }^{\otimes} \mathrm{CMV}$-positive tests (considering both IE-1 and pp65 markers) was consistently higher than that of pp65 alone, reaching $77.4 \%$ of positive tests around day 145 in D-/R+ patients (Online Supplementary Figure S4B). Thus, although generating lower spot counts, IE-1 antigen contributes significantly to T-Track ${ }^{\circledR} \mathrm{CMV}$ test positivity.

The patterns of SFC distribution relative to the start of the first CMV reactivation were comparable (Online Supplementary Figure S4C).

CMV-specific cell-mediated immunity measured after the end of treatment of a first CMV reactivation can predict recurrence of CMV reactivation

The primary aim of the study was to evaluate the suitability of measuring CMV-CMI after the end of treatment of a first-occurring CMV reactivation to predict freedom from or occurrence of a subsequent $\mathrm{CMV}$ reactivation (Figure 2A). CMV-CMI was measured on the day of discontinuation of antiviral treatment (day 0 ), and on days 7

Table 2. Cytomegalovirus reactivation according to the patients' cytomegalovirus serostatus.

\begin{tabular}{ccccc} 
& All & D- $/ \mathbf{R}+$ & $\mathbf{D}+/ \mathbf{R}+$ & $\mathbf{D +} / \mathbf{R}-$ \\
Study population, $\mathrm{n}$ & 154 & 82 & 53 & 19 \\
No documented CMV reactivation, $\mathrm{n}$ & 53 & 17 & 20 & 16 \\
At least one CMV reactivation, $\mathrm{n}$ & 101 & 65 & 33 & 3 \\
\hline One CMV reactivation only, $\mathrm{n}$ & 61 & 28 & 30 & 3 \\
Recurrent CMV reactivation, $\mathrm{n}$ & 40 & 37 & 3 & 0 \\
Two CMV reactivations, $\mathrm{n}$ & 24 & 21 & 3 & 0 \\
Three CMV reactivations, $\mathrm{n}$ & 16 & 16 & 0 & 0
\end{tabular}

CMV: cytomegalovirus; D-: CMV-negative donor; R+: CMV-positive recipient; D+: CMVpositive donor; R:- CMV-negative recipient. 
all patients

$\begin{array}{lccc}\mathrm{N}: & 65 & 29 & 10 \\ \text { Median VL: } & 2400 & 1800 & 830 \\ \text { (range): } & \left(100-1.8 \times 10^{6}\right) & \left(100-5.2 \times 10^{5}\right) & (100-7300)\end{array}$

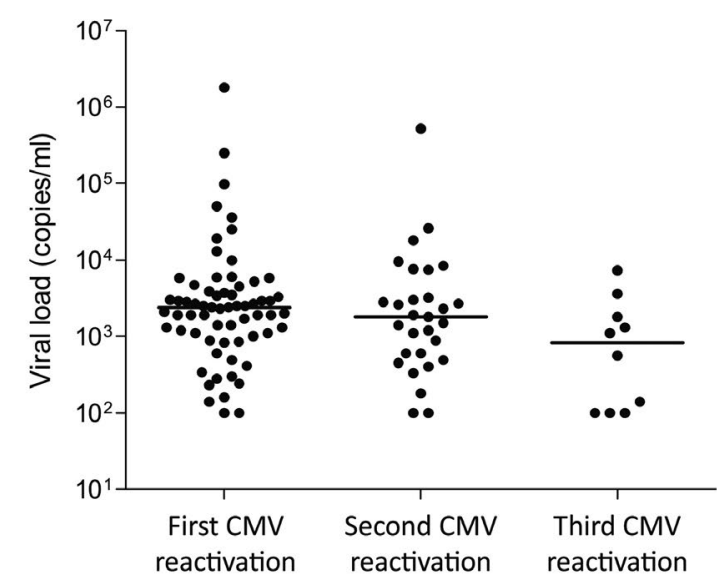

D-/R+ patients

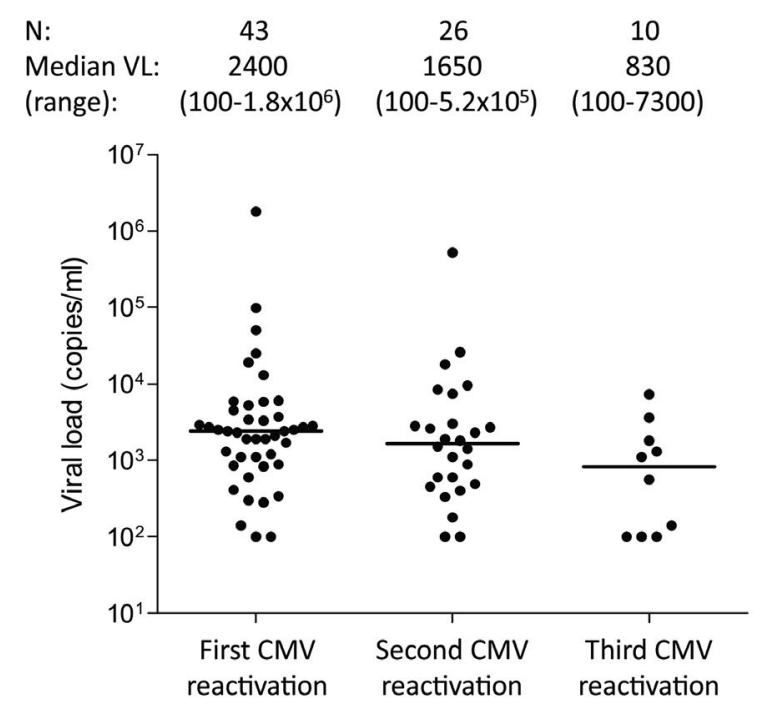

Figure 1. Cytomegalovirus (CMV) viral load at start of the first, second and third CMV reactivations. Median (range) CMV viral load (VL) at the time of the first, second and third CMV reactivations is shown for all patients and high-risk CMV-negative donor/CMV-positive recipient (D-/R+) pairs. It should be emphasized that VL measurements were not standardized among centers. CMV load was measured by quantitative polymerase chain reaction from whole blood ( 9 centers) or plasma ( 1 center) using either a commercial assay (Abbott RealTime CMV; 2 centers) or validated in-house protocols and equipment ( 8 centers). Accordingly, treatment-requiring viral load thresholds were center-specific and no analysis correlating spot-forming-cell counts to VL was planned in this study.

and 14 thereafter. The first available measurement was considered for the analysis. In fact, measurements on days 0,7 and 14 contributed 36/76 (47.4\%), 29/76 (38.1\%) and $11 / 76(14.5 \%)$ test results, respectively, to this analysis. SFC levels after a first CMV reactivation were compared between patients who experienced no further CMV reactivation and those with one or two subsequent (i.e., recurrent) CMV reactivations. A significant difference in SFC distribution was observed between the two groups, for both IE-1- and pp65-specific SFC levels, when considering all patients (Mann-Whitney $U$ test, $P<0.001$ ). The median SFC was, respectively, 10- and 40-times higher in patients free from recurrent $\mathrm{CMV}$ reactivation (based on squared mean of square-root-transformed [SRM^ ${ }^{\wedge}$ ] values) (Figure $2 \mathrm{~B})$. In the high-risk population, a significant difference was observed for pp65-induced response (Mann-Whitney $\mathrm{U}$ test, $P=0.001)$, but not for IE-1-mediated response (Mann-Whitney $U$ test, $P=0.724$ ) which was very low in both groups (Figure 2B). In accordance with these results, a ROC analysis in D-/R+ patients revealed AUC estimates significant for pp65-specific tests (AUC 0.780, 95\% confidence interval [95\% CI]: 0.642-0.917]; $P<0.001)$ but not for IE-1-specific tests (Figure 2C).

Interestingly, a post-hoc analysis of these data upon normalization of SFC values to absolute lymphocyte counts derived from peripheral blood counts (expressed as $\mathrm{SFC} / \mu \mathrm{L}$ blood) showed a stronger discrimination of SFC distributions between patients without and with recurrent $C M V$ reactivation, including in $\mathrm{D}-/ \mathrm{R}+$ patients (Mann-Whitney $U$ test, $P=0.010$ [IE-1] and $P<0.001$ [pp65]), and an improved predictive value in ROC analysis (AUC 0.760 [95\% CI: 0.599-0.921], $P=0.002$ for IE-1 and AUC 0.863 [95\% CI: 0.741-0.986], $P<0.001$ for pp65) (Online Supplementary Figure S5), compared to the normalization to 200,000 lymphocytes. SFC distributions according to conditioning regimen and $\mathrm{GvHD}$ occurrence are presented in Online Supplementary Figures S6 and S7, respectively.

The diagnostic accuracy of the ELISpot assay was determined in terms of sensitivity (patients with recurrent CMV reactivation had a negative test result after a first CMV reactivation) and specificity (patients free from recurrent $C M V$ reactivation had a positive test result after a first CMV reactivation) (Table 3 ). Likely due to the low SFC levels and high proportion of negative test results induced by IE-1, the IE-1-specific test alone showed limited performance in the $\mathrm{D}-/ \mathrm{R}+$ population (Table 3). By contrast, the performance of pp65-specific positive tests, alone or in combination with IE-1, to correctly identify patients free of future recurrent CMV reactivation was high, with a specificity of $77.8 \%$ (pp65) and $83.3 \%$ (pp65 and IE-1 combined) in D-/R+ patients (Table 3 ). The sensitivity of pp65-specific tests, alone or in combination with IE-1, in $\mathrm{D}-/ \mathrm{R}+$ patients was $58.6 \%$ and $55.2 \%$, respectively (Table 3 ). The positive predictive value (PPV; patients with a negative test after the first CMV reactivation had a subsequent recurrent CMV reactivation) of pp65-specific tests in $\mathrm{D}-/ \mathrm{R}+$ patients reached $80.9 \%$ (pp65 test alone) and $84.2 \%$ (pp65 and IE-1 tests combined) while the respective negative predictive values (NPV; patients with a positive test after the first CMV reactivation did not have a subsequent CMV reactivation) were low (53.8\% and 53.6\%, respectively) (Table 3 ).

The probability of recurrent CMV reactivation in patients with positive and negative ELISpot tests was estimated using Kaplan-Meier curves (Figure 2D). In line with the previous observations, the difference in probability of recurrent CMV reactivation between patients with a positive or negative pp65-specific test result after the first reactivation was highly significant, both in the total population (HR 4.91; log-rank test, $P<0.001)$ and in the $\mathrm{D}-/ \mathrm{R}+$ group (HR 2.52; log-rank test, $P=0.013$ ) (Figure 


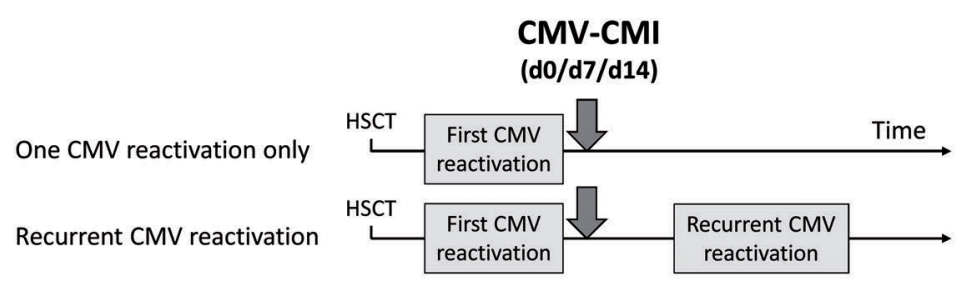

B

all patients

IE-1

Median (IQR): $13.9(2.0-52.4) \quad 1.3(0.1-6.2)$

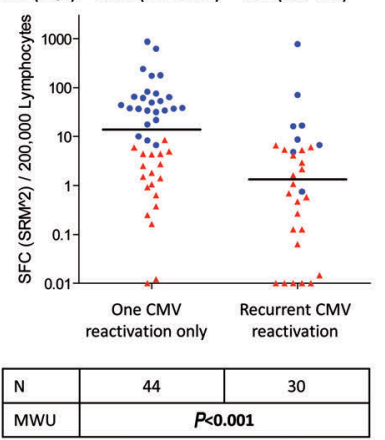

pp65

$155.8(29.5-595) \quad 3.6(0.1-15.9)$

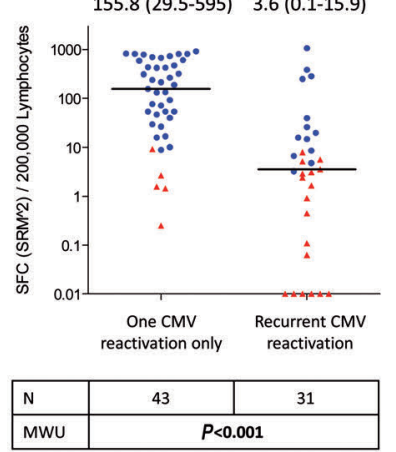

D-/R+

IE-1

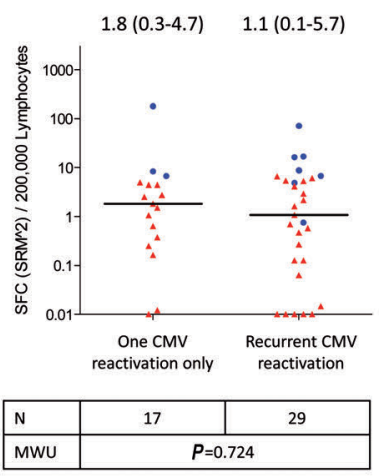

pp65

$36.7(9.1-153) \quad 3.6(0.1-15.3)$

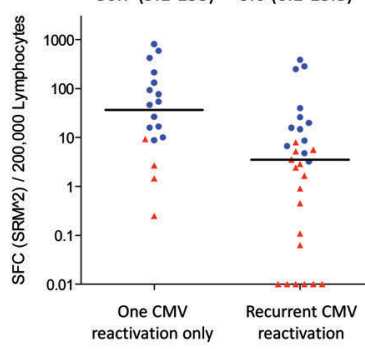

\begin{tabular}{|l|l|l|l|}
\hline $\mathrm{N}$ & 18 & 29 \\
\hline MWU & \multicolumn{3}{|c|}{$P=\mathbf{0 . 0 0 1}$} \\
\hline
\end{tabular}

C

all patients
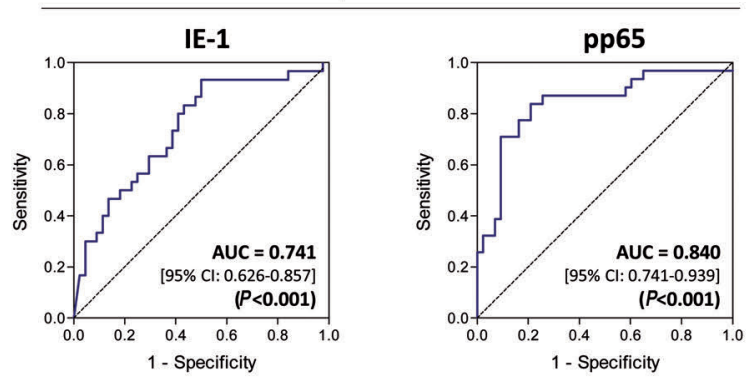

D-/R+

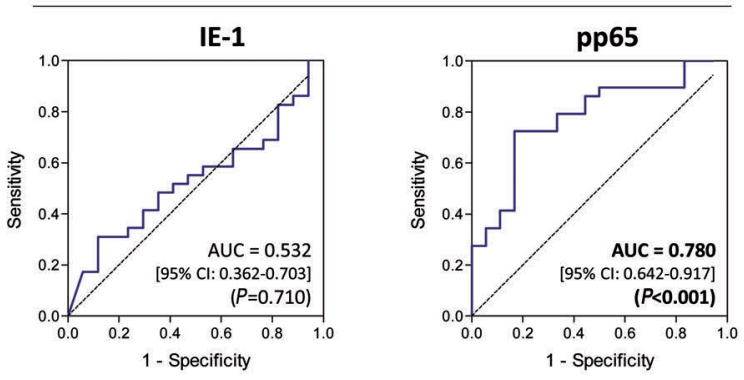

D

all patients
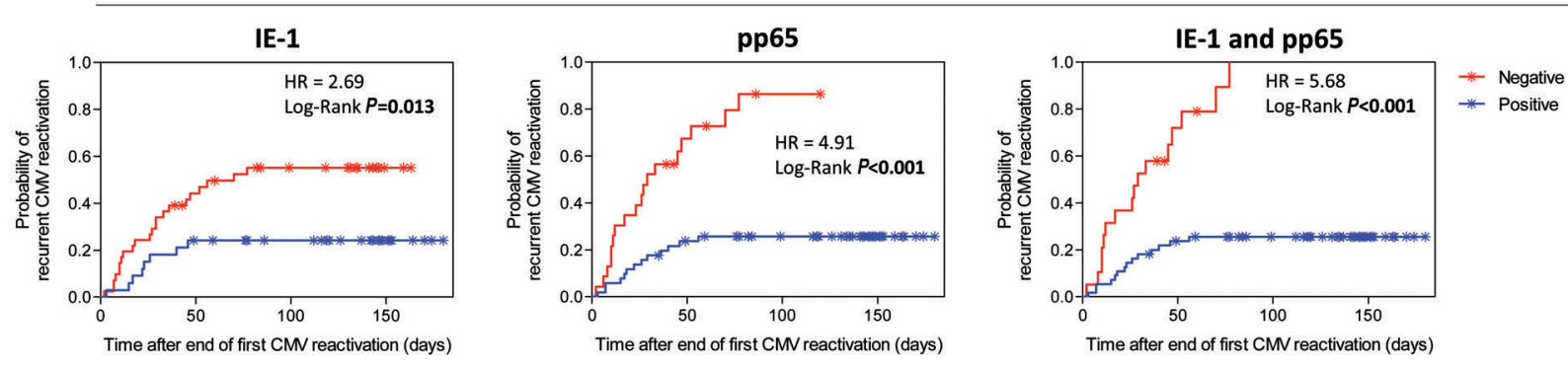

\section{D-/R+}
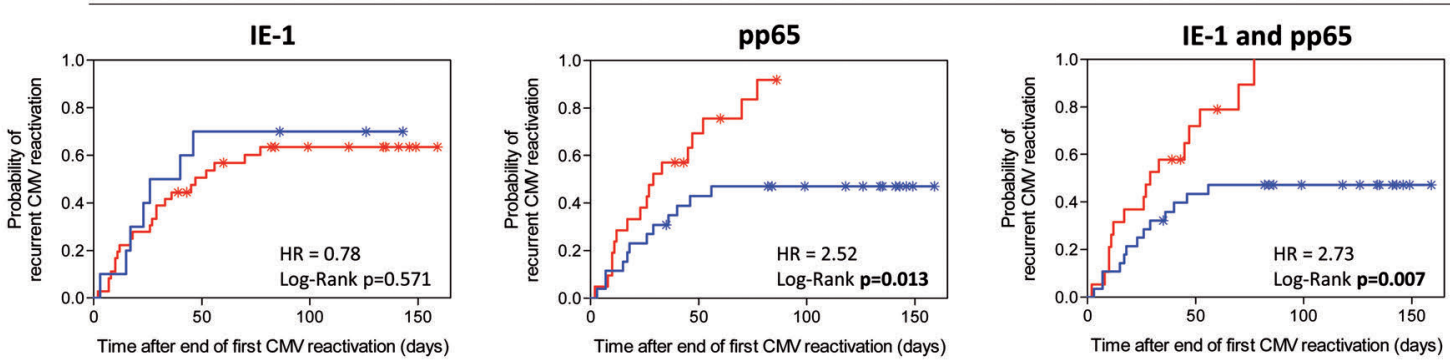
Figure 2 (previous page). Performance of cytomegalovirus (CMV)-specific cell-mediated immunity measured after the end of a first CMV reactivation to predict freedom from and/or occurrence of recurrent CMV reactivation. (A) Interferon- $\gamma$ enzyme-linked immunospot (ELISpot) was performed after the end of antiviral therapy for a first CMV reactivation, at up to three time points relative to the end of treatment, namely day 0 (d0), day 7 (d7) and day 14 (d14). The first available measurement was considered for the analysis. (B) Quantitative ELISpot results in response to CMV proteins IE- 1 and pp65 were evaluated on the basis of the mean of squareroot-transformed (SRM) spot-forming cells (SFC), as described in the Methods section. Differences in SFC distribution between patients with only one CMV reactivation and those with recurrent CMV reactivation were evaluated using a Mann-Whitney $U$ test. Respective $P$-values are shown under each graph. For the sake of simplicity, scatter plots are depicted as squared SRM values (SRM^2). The median and interquartile range of the SRM^2 SFC are shown above each graph. Additional information (minimum, maximum, $10^{\text {th }}$ and $90^{\text {th }}$ percentiles) are shown in Online Supplementary Table S3. Due to the log scale representation, values of zero SRM^ 2 were replaced by 0.01 (y-axis), meaning that baseline values shown at $y=0.01$ are actually equal to zero. Red triangles and blue dots represent negative and positive tests, respectively, defined according to the rules described in the Methods section. Of note, of the three CMV-negative donor/CMV-positive recipients (D-/R+) with a documented recurrent CMV reactivation and with high pp65-SFC after the first CMV reactivation (251 to 386 SFC/200,000 lymphocytes) one was treated for recurrent CMV although the viral load was below the center-specific threshold $(0$ or 100 copies $/ \mathrm{mL})$ in the 10 days preceding the start of treatment and at all time points thereafter; a second patient had a first treatment initiated for a viral load below the center-specific threshold, after which high pp65-specific SFC dropped dramatically over time before the start of treatment of a CMV reactivation with a viral load above the threshold; the third patient had a lengthy ( $>3$ months) first CMV reactivation with a high sustained viral load (up to 110,000 copies/mL), likely reflecting refractory CMV. ${ }^{55}$ Importantly, the duration of antiviral therapy for a first CMV reactivation was comparable in patients without and with recurrent reactivation (median [range] duration of 25 [4-94] and 31 [3-77] days, respectively, in all patients [Mann-Whitney $U$ test, $P=0.336$ ]; median [range] duration of 31 [4-94] and 34 [7-77] days, respectively, in $\mathrm{D}-/ \mathrm{R}+$ ptients [Mann-Whitney $\mathrm{U}$ test, $P=0.677$ ]). (C) Prediction of CMV reactivation recurrence based on IE-1- and pp65-specific SFC counts measured at the end of treatment of a first CMV reactivation was evaluated by receiver operating characteristic curve analysis. Area under the curve estimates, $95 \%$ confidence intervals and respective $P$-values are indicated within each graph. (D) Cumulative probability of CMV reactivation recurrence based on IE-1- and pp65-specific qualitative test results after a first CMV reactivation, evaluated as described in the Methods section. In the case that both IE-1 and pp65 test results are considered (T-Track ${ }^{\circledR}$ CMV; right panels), a test is positive when at least one IE-1 and/or pp65 test is positive and a test is negative when both IE-1 and pp65 tests are negative. Kaplan-Meier analyses were performed and the respective hazard ratios and $P$-values are shown within each graph. $* / *$ indicate censored observations. The median (range) follow-up time after the T-Track ${ }^{\circledast} \mathrm{CMV}$ measurement was 137 (35-180) days in patients with no documented recurrent CMV reactivation (censored). The median (range) time to recurrent CMV reactivation after the T-Track CMV measurement was 24 (2-77) days. Moreover, the last recurrent CMV event in the case of a pp65- (and T-Track ${ }^{\circledR}$ CMV)-positive test result occurred 56 days after the end of antiviral therapy, compared to 77 days in the case of a pp65- and IE-1-negative test result. In (B-D), statistically significant $P$-values are in bold. CMI: cellmediated immunity; HSCT: hematopoietic stem cell transplantation; IQR: interquartile range; MWU: Mann-Whitney U test; D-/R+: CMV-negative donor/CMV-positive recipient; AUC: area under curve; 95\% Cl: 95\% confidence interval; HR: hazard ratio.

Table 3. Diagnostic accuracy in identifying patients with and without recurrent cytomegalovirus (CMV) reactivation based on CMV-specific negative and positive enzyme-linked immunospot test results after the first CMV reactivation.

\begin{tabular}{|c|c|c|c|c|c|c|}
\hline Population & Marker & Sensitivity & Specificity & Chi-square & PPV & $N P V^{b}$ \\
\hline \multirow{3}{*}{ All patients } & IE-1 & $\begin{array}{c}73.3 \%(22 / 30) \\
\text { [95\% CI: } 54.1-87.7 \%]\end{array}$ & $\begin{array}{c}56.8 \%(25 / 44) \\
\text { [95\% CI: 41.0-71.7\%] }\end{array}$ & $P=0.010$ & - & - \\
\hline & pp65 & $\begin{array}{c}58.1 \%(18 / 31) \\
\text { [95\% CI: 39.1-75.5\%] }\end{array}$ & $\begin{array}{c}88.4 \%(38 / 43) \\
\text { [95\% Cl: } 74.9-96.1 \%]\end{array}$ & $P<0.001$ & - & - \\
\hline & IE-1, pp65a & $\begin{array}{c}53.3 \%(16 / 30) \\
\text { [95\% CI: 34.3-71.7\%] }\end{array}$ & $\begin{array}{c}93.2 \%(41 / 44) \\
\text { [95\% CI: 81.3-98.6\%] }\end{array}$ & $P<0.001$ & - & - \\
\hline $\mathrm{D}-/ \mathrm{R}+$ patients & $\begin{array}{c}\text { IE-1 } \\
\text { pp65 } \\
\text { IE-1, pp65 }\end{array}$ & $\begin{array}{c}75.9 \%(22 / 29) \\
\text { [95\% CI: } 56.5-89.7 \%] \\
58.6 \%(17 / 29) \\
\text { [95\% CI: } 38.9-76.5 \%] \\
55.2 \%(16 / 29) \\
\text { [95\% CI: } 35.7-73.6 \%]\end{array}$ & $\begin{array}{c}17.7 \%(3 / 17) \\
\text { [95\% CI: } 3.8-43.4 \%] \\
77.8 \%(14 / 18) \\
\text { [95\% CI: } 52.4-93.6 \%] \\
83.3 \%(15 / 18) \\
\text { [95\% CI: } 58.6-96.4 \%]\end{array}$ & $\begin{array}{l}P=0.606 \\
P=0.015 \\
P=0.009\end{array}$ & $\begin{array}{c}61.1 \% \\
(22 / 36) \\
80.9 \% \\
(17 / 21) \\
84.2 \% \\
(16 / 19)\end{array}$ & $\begin{array}{c}30.0 \% \\
(3 / 10) \\
53.8 \% \\
(14 / 26) \\
53.6 \% \\
(15 / 28)\end{array}$ \\
\hline
\end{tabular}

${ }^{a}$ T-Track ${ }^{\oplus} \mathrm{CMV}$ assay: the test is positive when at least one of the IE-1- and/or pp65-specific response is positive, and the test is negative when both IE-1-and pp65-specific response are negative; 'Positive and negative predictive values were not calculated in the "all patients" population because of the imbalance toward CMV-negative donor/CMV-positive recipient patients in that group. 95\% CI: 95\% confidence interval; CMV: cytomegalovirus; D/R: donor/recipient CMV serostatus; NPV: negative predictive value; PPV: positive predictive value.

2D). Interestingly, the performance of the pp65 test was improved by the combination with IE-1 (T-Track ${ }^{\circledR} \mathrm{CMV}$ test), in all patients (HR 5.68; log-rank test, $P<0.001$ ) and in $\mathrm{D}-/ \mathrm{R}+$ patients (HR 2.73; log-rank test, $P=0.007$ ) (Figure 2D).

To better understand the usability of the assay in terms of clinical cutoff, we evaluated the PPV (patients with SFC $\leq$ threshold after the first CMV reactivation had a recurrent CMV reactivation) and NPV (patients with $\mathrm{SFC}>$ threshold after the first CMV reactivation did not have a recurrent CMV reactivation) of pp65-specific response in $\mathrm{D}-/ \mathrm{R}+$ patients at low, intermediate and high SFC counts (Table 4). A NPV of $100 \%$ (3/3) was observed for a threshold of 386 SFC/200,000 lymphocytes in association with a PPV of $65.9 \%(29 / 44)$. A NPV of $75.0 \%(9 / 12)$ and PPV of $74.3 \%(26 / 35)$ were found for a SFC of 40 SFC/200,000 lymphocytes. Higher PPV (87.5\% [21/24] to $100 \%$ [8/8]) and lower NPV (65.2\% [15/23] to 46.2\% [18/39]) were observed at lower ( 9 and less) SFC counts (Table 4), in line with the results described above (Table 3 ).

\section{Benefit of monitoring CMV-specific cell-mediated immunity over absolute T-cell counts}

We next compared the performance of CMV-CMI to that of absolute lymphocyte ( $\mathrm{T}$ and NK cells) counts measured after end of treatment of a first CMV reactivation to predict subsequent CMV reactivation episodes. Multicolor flow cytometry was performed using remaining peripheral blood mononuclear cells. NK and T (total, naive and memory) cell levels were expressed as absolute cell counts. The first visit with existing absolute cell counts following the end of antiviral therapy (of day 0,7 and 14) was considered for the analysis. Absolute cell counts were significantly higher in patients with no recurrent CMV reactivation in all cases, except for total and memory $\mathrm{CD}^{+} \mathrm{T}$ cells in $\mathrm{D}-/ \mathrm{R}+$ patients (Online Supplementary Figure S8A). In ROC analyses, total lymphocytes as well as total and naive $\mathrm{CD}^{+}$cell populations showed a good predictive value for recurrent CMV reactivation, with AUC estimates between 0.813 and 0.833 in $\mathrm{D}-/ \mathrm{R}+$ patients (Online Supplementary Figure S8B), thus in a 
Table 4. Positive and negative predictive values of low, intermediate and high spot-forming-cell counts of a pp65-specific enzyme-linked immunospot assay after the end of treatment for a first cytomegalovirus (CMV) reactivation to predict the occurrence of future recurrent CMV reactivation in high-risk hematopoietic stem cell transplant patients.

\begin{tabular}{lcccccccc} 
Patient population & Marker & Threshold & \multicolumn{2}{c}{ PPV } & \multicolumn{2}{c}{ NPV } & \% patients above threshold \\
& & 386 & $65.9 \%$ & $(29 / 44)$ & $100 \%$ & $(3 / 3)$ & $6.4 \%$ & $(3 / 47)$ \\
D-/R+ & & 40 & $74.3 \%$ & $(26 / 35)$ & $75.0 \%$ & $(9 / 12)$ & $25.5 \%$ & $(12 / 47)$ \\
& \multirow{3}{*}{ pp65 } & 9 & $87.5 \%$ & $(21 / 24)$ & $65.2 \%$ & $(15 / 23)$ & $48.9 \%$ & $(23 / 47)$ \\
& & 0 & $100 \%$ & $(8 / 8)$ & $46.2 \%$ & $(18 / 39)$ & $83.0 \%$ & $(39 / 47)$ \\
\hline
\end{tabular}

aSF (SRM^2)/200,000 lymphocytes (stimulated minus unstimulated condition) rounded to closest spot count; thresholds were derived from Receiver operating characteristic curve data; the threshold of $9 \mathrm{SFC}\left(\mathrm{SRM}^{\wedge} 2\right) / 200,000$ lymphocytes showed the highest sensitivity+specificity value. SFC: spot-forming cells; $\mathrm{SRM}^{\wedge} 2$ : squared mean of square-roottransformed; CMV;: cytomegalovirus; D/R: donor/recipient CMV serostatus; NPV: negative predictive value; PPV: positive predictive value.

comparable range to that of the AUC derived from pp65specific ELISpot test results (Figure 2C and Online Supplementary Figure S5C). NK cells as well as total, naive and memory $\mathrm{CD}^{+} \mathrm{T}$-cell subpopulations showed a lower predictive value, especially in $\mathrm{D}-/ \mathrm{R}+$ patients, with AUC estimates between 0.615 and 0.729 (Online Supplementary Figure S8B). A ROC analysis of paired pp65-specific ELISpot results and absolute lymphocyte and T-cell counts obtained at the same visit after the end of therapy showed no statistically significant difference in predictability, although pp65-specific ELISpot results normalized to absolute lymphocyte counts tended to perform better than absolute cell counts (Online Supplementary Figure S8C).

\section{CMV-specific cell-mediated immunity at day 100 post-transplantation can predict late recurrent CMV reactivation (post-hoc analysis)}

Accurate prediction of future recurrent CMV reactivation is particularly critical when patients are released from close monitoring in an outpatient setting around 3 months after HSCT. ${ }^{46}$ We conducted two post-hoc analyses to determine whether CMV-CMI monitoring at the fixed time of day 100 could identify patients at risk of future (i.e. late) CMV reactivation. The first analysis considered all patients, regardless of a possible existence of CMV reactivation prior to day 100 , thus assessing occurrence of late CMV reactivation generally (Online Supplementary Figure S9). The second analysis focused on patients who experienced CMV reactivation prior to day 100 , thus investigating the usefulness of the IFN- $\gamma$ ELISpot measured around day 100 to predict late recurrent CMV reactivation (Figure 3). T-Track ${ }^{\circledast} \mathrm{CMV}$ test results acquired between day 80 and day 100 after transplantation were considered in patients with no ongoing CMV reactivation (Online Supplementary Methods).

Interestingly, patients (including those in the high-risk group) who did not experience CMV reactivation up to day 80-100 did not experience CMV reactivation thereafter (Online Supplementary Figure S9B, orange-labeled dots). A majority of these patients presented low IE-1 and pp65-specific test results throughout the study (Online Supplementary Figure S9B and data not shown). Such sustained low responsiveness in patients with no CMV reactivation was previously reported. ${ }^{33}$ Its cause remains to be investigated. Consequently, performance of the ELISpot test at day 100 in this global analysis of late CMV reactivation was low (Online Supplementary Figure S9B-D; Online Supplementary Table S5). On the other hand, the analysis of ELISpot test results at day 100 in patients with an earlier CMV reactivation (Figure $3 \mathrm{~A}$ ) revealed a significant difference in SFC counts between patients without and with late recurrent $\mathrm{CMV}$ reactivation (Mann-Whitney $\mathrm{U}$ test, $P$ value between 0.013 and $<0.001)$, with the exception of IE-1-specific response in D-/R+ patients (Mann-Whitney $\mathrm{U}$ test, $P=0.277$ ) (Figure $3 \mathrm{~B}$ ). All patients with late recurrent $\mathrm{CMV}$ reactivation belonged to the high-risk group. In ROC analyses, AUC estimates for pp65 test results were $0.811(P=0.003)$ in the $\mathrm{D}-/ \mathrm{R}+$ population and 0.911 $(P<0.001)$ in the total population (Figure 3C). pp65-specific response showed the best diagnostic accuracy, with a sensitivity and specificity of $75.0 \%$ and $90.9 \%$, and $\mathrm{PPV}$ and NPV of $90.0 \%$ and $76.9 \%$ in the D-/R+ population, respectively (Table 5). The probability of late recurrent CMV reactivation was significantly higher in D-/R+ patients with a pp65-negative test at day 100 (HR 6.34; log-rank test, $P=0.002)$. In this setting, the response to IE1 did not improve the performance of pp65 (Figure 3D). Remarkably, the probability of recurrent CMV in the case of pp65- and T-Track ${ }^{\circledR} \mathrm{CMV}$-positive tests was lower when measured at day 100 than when measured directly after a first CMV reactivation (compare blue curves in Figures $2 \mathrm{D}$ and $3 \mathrm{D}$ ).

\section{Discussion}

This study demonstrates for the first time the suitability of a standardized CMV-specific IFN- $\gamma$ ELISpot assay to predict recurrence of CMV reactivation after HSCT, with a particular focus on clinically relevant high-risk $\mathrm{D}-/ \mathrm{R}+$ patients.

Overall, the response to pp65 antigen in the ELISpot assay was higher than that of IE-1, as previously reported. $^{26,27,30}$ Although the IE-1-specific response had low or no predictive value alone, it improved the assay performance in combination with pp65-specific tests after the first CMV reactivation.

Diagnostic accuracy and time-to-event analyses indicated that negative IFN- $\gamma$ ELISpot test results could predict recurrence of CMV reactivation when measured after the end of anti-CMV therapy or around day 100 after HSCT. Positivity of the T-Track ${ }^{\circledR}$ CMV test is based on several rules, the main one being that $\mathrm{CMV}$ antigen-stimulated conditions must yield $\geq 10$ SFC (or mean of sqrt SFC $\geq 3.16$ ) (Online Supplementary Methods). This raises the possibility that the technical cutoff of the assay might be a relevant clinical cutoff for the prediction of recurrent CMV reactivation (PPV $>80 \%)$. Whether the positivity cutoff of $\mathrm{T}$ Track $^{\otimes}$ CMV is indeed a valid clinical cutoff to predict recurrent CMV reactivation in high-risk D-/R+ patients could be addressed in a randomized interventional study. In fact, such an approach is currently being appraised in high-risk solid-organ transplant recipients in a study aim- 
A

CMV-CMI

d80-d100 post-HSCT
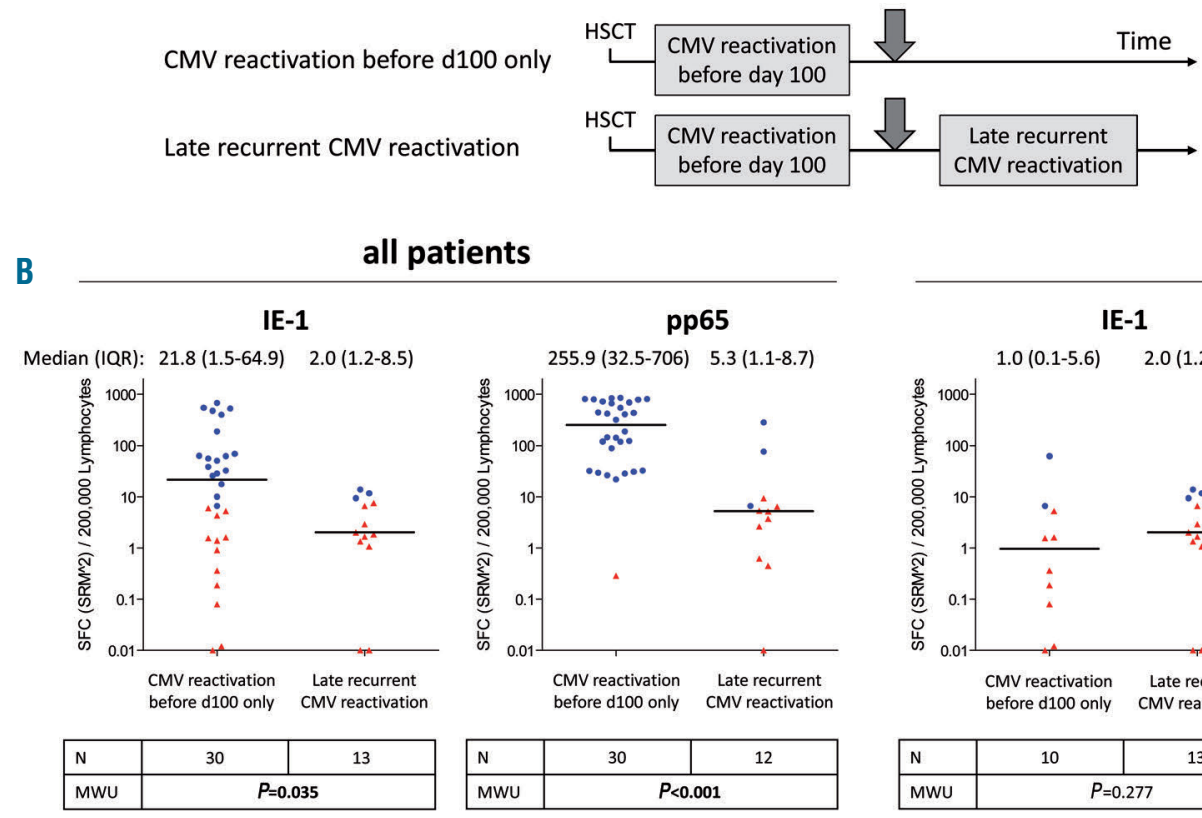

all patients

pp65

$255.9(32.5-706) \quad 5.3(1.1-8.7)$

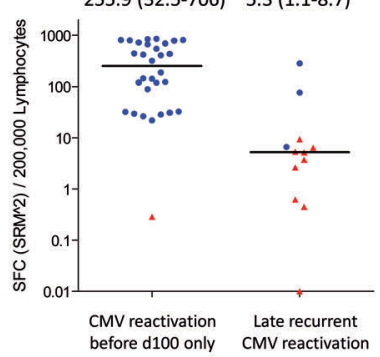

\begin{tabular}{|l|l|l|l|}
\hline $\mathrm{N}$ & 30 & \multicolumn{1}{|c|}{12} \\
\hline MWU & \multicolumn{3}{|c|}{$P<0.001$} \\
\hline
\end{tabular}

D-/R+

IE-1

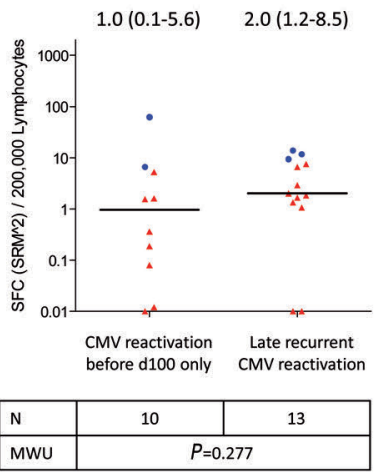

pp65

$32.1(26.5-142) \quad 5.3(1.1-8.7)$

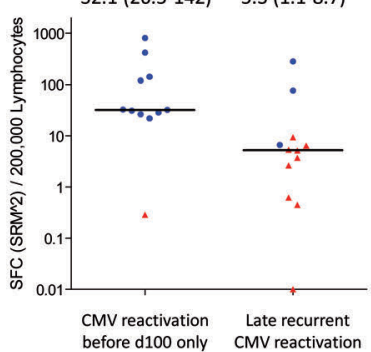

C

\section{all patients}

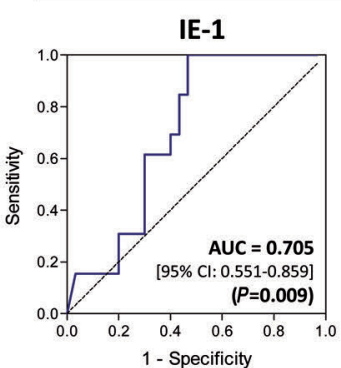

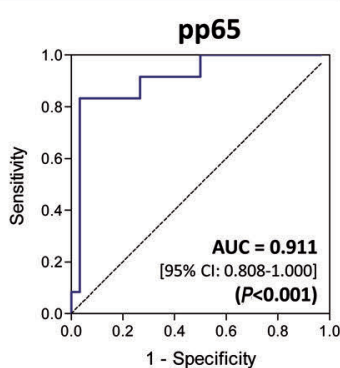

D-/R+

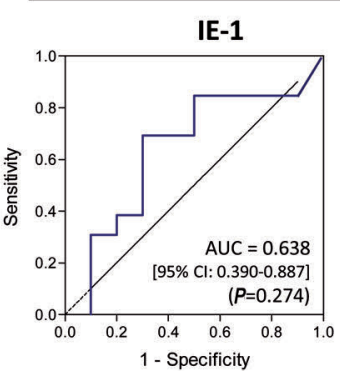

\begin{tabular}{|l|l|l|l|}
\hline N & 11 & \multicolumn{1}{|c|}{12} \\
\hline MWU & \multicolumn{4}{|c|}{$P=0.013$} \\
\hline
\end{tabular}

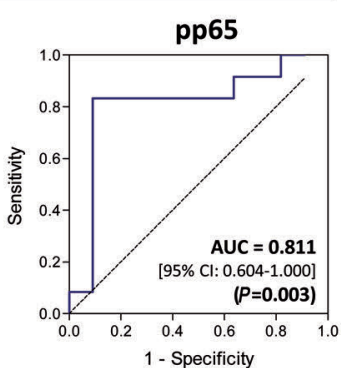

D

\section{all patients}
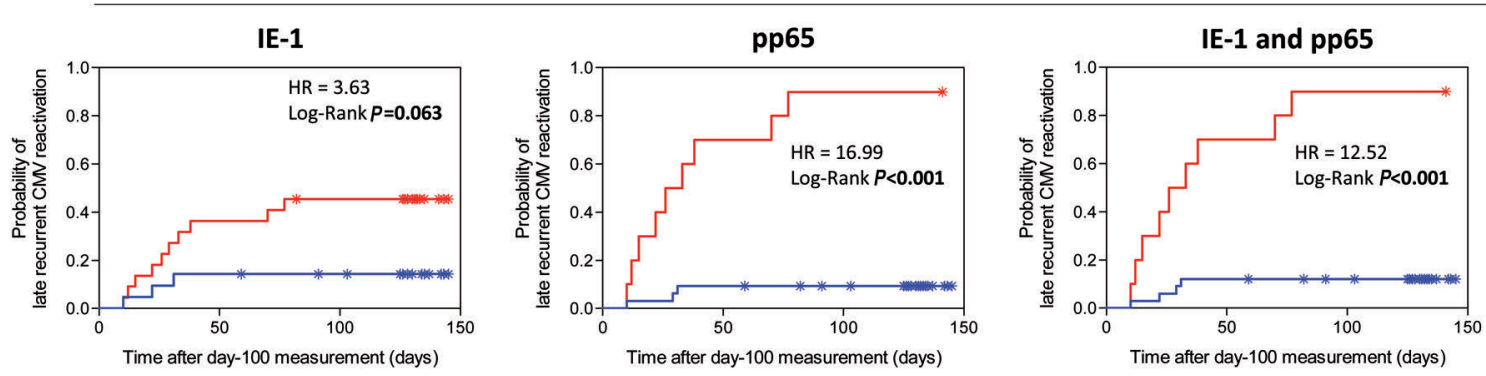

* Negative

* Positive

\section{D-/R+}
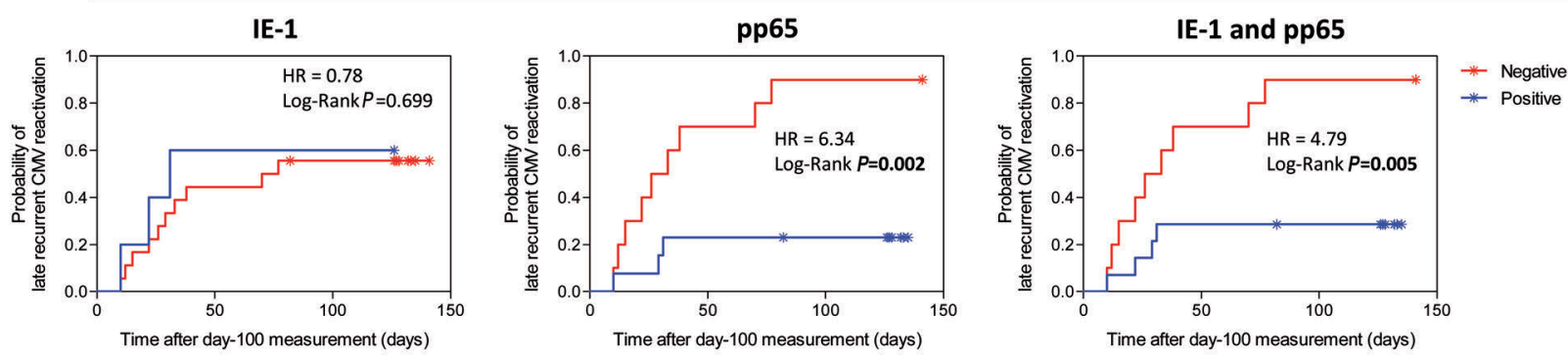

Figure 3. Legend on following page. 
Figure 3 (previous page). Performance of cytomegalovirus (CMV) cell-mediated immunity measured around day 100 after hematopoietic stem cell transplantation to predict freedom from and occurrence of late recurrent CMV reactivation (post-hoc). (A) Interferon-y enzyme-linked immunospot (ELISpot) tests performed between day $80(\mathrm{~d} 80)$ and day $100(\mathrm{~d} 100)$ after hematopoietic stem cell transplantation in patients with an earlier CMV reactivation were used for the analysis. In the case that several measurement were available, the one closest to day 100 was considered. (B) Quantitative ELISpot results in response to CMV IE-1 and pp65 proteins were evaluated as detailed in the legend to Figure 2. Differences in spot-forming cell (SFC) distribution between patients with a CMV reactivation before day 100 only and those with a late (after day 100) recurrent CMV reactivation were evaluated using a Mann-Whitney $\mathrm{U}$ test. The median and interquartile range of the squared mean of square-root-transformed $\left(\mathrm{SRM}^{\wedge} 2\right) \mathrm{SFC}$ are shown above each graph. Additional information (minimum, maximum, $10^{\text {th }}$ and $90^{\text {th }}$ percentiles) are shown in Online Supplementary Table S4. Due to the log scale representation, values of zero SRM^ 2 were replaced by 0.01 ( $y$-axis), meaning that baseline values shown at $y=0.01$ are actually equal to zero. Red triangles and blue dots represent negative and positive tests, respectively, defined according to the rules described in the Online Supplementary Methods. (C) Prediction of late recurrent CMV reactivation based on IE-1- and pp65-specific SFC counts measured around day 100 was evaluated by receiver operating characteristic curve analysis, as detailed in the legend to Figure 2. (D) Cumulative incidence of recurrent CMV reactivation based on IE1- and pp65-specific qualitative test results around day 100, was evaluated as detailed in the legend to Figure 2. Median (range) follow up time after T-Track ${ }^{\circledR}$ CMV measurement was 132 (59-145) days in (censored) patients with no documented late recurrent CMV reactivation. The median (range) time to late recurrent CMV reactivation after T-Track ${ }^{\circledast}$ CMV measurement was 119 (105-165) days. Moreover, the last recurrent CMV event in the case of a pp65- (and T-Track ${ }^{\circledast}$ CMV)-positive test result occurred 31 days after the day-100 measurement, compared to 77 days in the case of a pp65- and IE-1-negative test result. CMI: cell-mediated immunity; HSCT: hematopoietic stem cell transplantation; IQR: interquartile range; MWU: Mann-Whitney U test; D-/R+: CMV-negative donor/CMV-positive recipient; AUC: area under curve; 95\% Cl: 95\% confidence interval; HR: hazard ratio.

Table 5. Diagnostic accuracy in identifying patients with and without late (beyond day 100 after hematopoietic stem cell transplantation) recurrent cytomegalovirus (CMV) reactivation based on CMV-specific negative and positive enzyme-linked immunospot test results at day 80 to 100 .

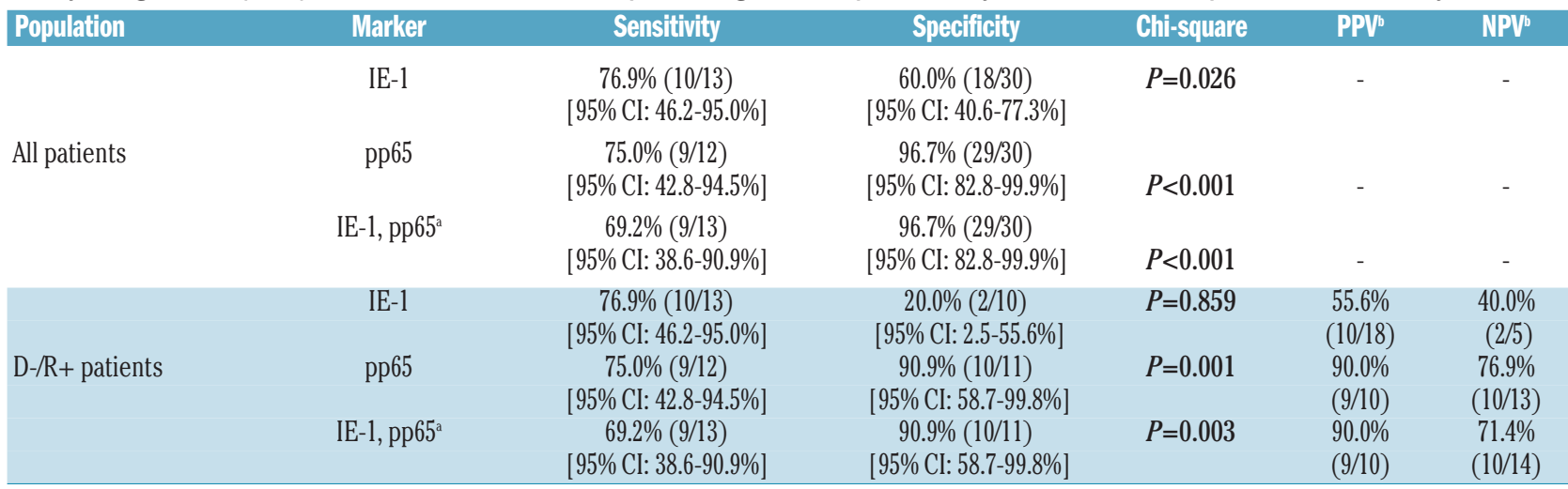

${ }^{a}$ T-Track ${ }^{\circledR}$ CMV assay: test is positive when at least one of the IE-1- and/or pp65-specific response is positive, and the test is negative when both IE-1- and pp65-specific responses

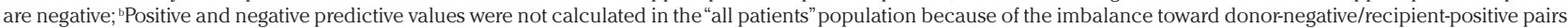
in that group. $95 \%$ CI, $95 \%$ confidence interval; CMV, cytomegalovirus; D/R, donor/recipient CMV serostatus; NPV, negative predictive value; PPV, positive predictive value.

ing to steer the duration of antiviral prophylaxis based on CMV-CMI monitoring using T-Track ${ }^{\circledR}$ CMV (ClinicalTrials.gov Identifier: NCT02538172).

The finding that the IFN- $\gamma$ ELISpot assay measured after a first CMV reactivation performs at least as well as the measurement of absolute lymphocyte subset counts to predict subsequent $\mathrm{CMV}$ reactivation offers the attractive possibility of introducing T-Track ${ }^{\circledR} \mathrm{CMV}$ in complement to the currently implemented absolute T-cell count measurement to improve risk stratification of recurrent CMV after HSCT. The value of absolute lymphocyte counts ${ }^{13,34,35}$ and of absolute lymphocyte subsets ${ }^{15,17,32,36-40}$ to predict $\mathrm{CMV}$ infection and/or recurrence is well documented, in line with the described protective role of $\mathrm{CD}^{+}, \mathrm{CD}^{+}, \gamma \delta$ $\mathrm{T}$ and NK cells against CMV reactivation..$^{15,40-48}$ In general, absolute T-cell counts recovery correlates well with CMVspecific T-cell reconstitution in vivo, ${ }^{17,32,47,49}$ and absolute lymphocyte, $\mathrm{CD}^{+}$and/or $\mathrm{CD}^{+}{ }^{+} \mathrm{T}$-cell counts have been proposed as surrogate markers for CMV-specific cellular immunity. ${ }^{34,35,47}$ The good predictive value of absolute lymphocyte counts probably explains the improved performance of the ELISpot assay observed upon normalization to absolute lymphocytes, which might be a relevant normalization method in HSCT patients.

On the other hand, absolute T-cell counts do not always reflect $C M V$-specific immune reconstitution. ${ }^{49,50}$ Recovery of CMV-specific T cells is often delayed relative to that of bulk T cells, especially in patients with recurrent CMV reactivation. ${ }^{49}$ Moreover, dysfunctional $\mathrm{CD}^{+} \mathrm{T}$ cells spe- cific for CMV accumulate in patients with recurrent CMV reactivation. ${ }^{51}$ Thus, functionality (i.e., the capacity of cells to respond to antigen stimulation) rather than the number of $\mathrm{T}$ cells is more likely to accurately reflect protection against recurrent $\mathrm{CMV}$ reactivation. Accordingly, we observed several cases of discordant absolute CD8 ${ }^{+} \mathrm{T}$ cell counts and IFN- $\gamma$ ELISpot measurements in favor of the ELISpot assay, in particular cases of high $\mathrm{CD}^{+} \mathrm{T}$-cell count together with low T-Track ${ }^{\circledR}$ CMV results prior to or during CMV reactivation episodes (data not shown). Therefore, CMV-specific immune monitoring using a standardized assay, while complementing the current approach of absolute T-cell count monitoring, provides an additional value for the risk stratification of recurrent CMV reactivation in HSCT patients.

Negative ELISpot test results after the first CMV reactivation and at day 100 predicted subsequent recurrent CMV reactivation with a PPV of $80 \%$ and $90 \%$, respectively. Measuring pp65-specific response at day 100 rather than directly after the end of a first CMV reactivation also improved the NPV (77\% vs. 54\%, respectively). This suggests that measuring CMV-CMI at the fixed time point of day 100 might be of greater clinical relevance to predict recurrence of CMV. The apparent improvement in NPV (and PPV) of the day-100 measurement might be in part due to the overall later measurement time (median [range] time of 93 [80-100] days), compared to the time of measurement at the end of treatment for a first CMV reactivation, which ranged from day 45 to 186 after transplanta- 
tion (median 82 days). Moreover, most (31/44 [70.4\%]) of the day-100 measurements took place 14 days or later relative to the end of a first antiviral treatment, while most (36/76 [47.4\%]) of the measurements at the end of a first CMV reactivation were from the day of discontinuation of antiviral treatment (day 0). This raises the possibility that measuring CMV-CMI 14 days after the end of a treatment-requiring CMV reactivation - rather than on the day of antiviral treatment discontinuation - might improve the predictive value for protection against recurrent reactivation. This might be explained by the ongoing immune reconstitution that takes place after HSCT. Indeed, a paired comparison of existing day 0 , day 7 and day 14 measurements revealed a statistically significant increase in SFC counts between day 7 (or day 0) and day 14 in patients with no future recurrent CMV reactivation (data not shown). A future study should determine whether measuring CMV-CMI 14 days after the end of antiviral treatment and/or whether monitoring the dynamics of response might improve prediction of recurrent CMV reactivation.

While the current focus on high-risk (D-/R+) HSCT patients for the prediction of recurrent CMV reactivation is of major clinical relevance, it constitutes the main limitation of this study. A recent study in intermediate-risk $(\mathrm{D}+\mathrm{R}+)$ HSCT patients partly addressed this question by measuring the dynamic changes in CMV-CMI using TTrack $^{\circledR} \mathrm{CMV}^{52}$ underscoring the usefulness of multiple measurements for risk stratification of HSCT recipients. It should also be emphasized that despite the use of nonstandardized conditioning regimens, viral load testing and treatment protocols in this multicenter study, the IFN- $\gamma$ ELISpot assay performed very well at predicting recurrent $\mathrm{CMV}$ reactivation. This further highlights the applicability of using a sensitive and standardized CMV-CMI monitoring assay in a real-world setting, as well as its ease of implementation in clinical routine, for the risk stratification of HSCT patients.

In conclusion, our results suggest that using a standardized CMV-CMI monitoring assay can support clinicians in the identification and management of patients with increased risk of recurrent CMV reactivation following HSCT. Beside its applicability in the preemptive setting, the door is also open to its implementation in novel clinical settings with unmet medical needs, such as steering the duration of antiviral prophylaxis (e.g., using letermovir $)^{53}$ and monitoring immune reconstitution following adoptive $\mathrm{T}$-cell transfer. ${ }^{54}$

\section{Disclosures}

The participating clinical and measurement centers (D.W., $E W, D T, C W, D J, K S-E, J G, S M, M S, G K, M K, I H, M L-T$, $S K, D H, S K, M V, S G, M D, T G, M K, T H, M L)$ received research funding from Lophius Biosciences for this study. $L D$ is an employee, co-founder, Chief Scientific Officer and shareholder of Lophius Biosciences. SB is an employee and shareholder of Lophius Biosciences. TS, H.G. and A.R. are employees of Lophius Biosciences. RW is Chairman of the Board and a shareholder of Lophius Biosciences. Issued patents relevant to the TTrack $\AA$ CMV assay used in this study: WO/2010/115984 "METHOD FOR POLYPEPTIDE TRANSFER INTO CELLS". WO/2003/080792 "USE OF UREA-ADJUVATED POLYPEPTIDES FOR DIAGNOSIS, PROPHYLAXIS AND TREATMENT". WO/2003/046212 "METHOD FOR IDENTIFYING TARGET EPITOPES OF THE T CELL MEDIATED IMMUNE RESPONSE AND FOR ASSAYING EPITOPE-SPECIFIC T CELLS"

\section{Contributions}

$L D, D W, S B$ and $R W$ contributed to the study conception and design; TS, LD, SB and RW organized, coordinated and supervised the study and its logistics; DW, EW, DT, CW, DJ, KS-E, $J G, S M, M S, G K, M K, I H, M L-T, S K, D H, S K, M V, S G$, $M D$ and TG acquired patient samples and contributed to data collection; EW, TH, M.K., M.L. and S.B. performed the ELISpot assays; SB and HG analyzed the ELISpot results; $A R$, $T S, L D$ and $R W$ conducted and supervised the interpretation of results; $A R$ drafted the manuscript and figures; All authors contributed to, reviewed and approved the manuscript.

\section{Acknowledgments}

This study was supported in part by the German Federal Ministry of Education and Research (BMBF grant number 031A215) to Lophius Biosciences and by research funding from Lophius Biosciences to DW, EW, DT, CW, DJ, KS-E, JG, SM, $M S, G K, M K, I H, M L-T, S K, D H, S K, M V, S G, M D, T G$, $M K, T H$, and ML. DW was supported by the Deutsche Forschungsgemeinschaft (DFG, German Research Foundation, grant number 324392634 - TRR 221). We are grateful to all study participants for their contribution. The authors acknowledge the members of the respective participating centers, as well as Anja Svenson, Julia Batzilla, Hanna Bendfeldt, Astrid Starke and Alcedis GmbH (Giessen, Germany) for their expertise and assistance in data collection, data management, and study planning and coordination. We thank Thomas Keller and Stephan Weber (ACOMED Statistik, Leipzig, Germany) for their statistical evaluation. T-Track ${ }^{\circledR} C M V$ tests were provided by Lophius Biosciences GmbH, Regensburg, Germany.

\section{Funding}

This study was supported in part by the German Federal Ministry of Education and Research (BMBF grant number 031A215) to Lophius Biosciences and by research funding from Lophius Biosciences to DW, EW, DT, CW, DJ, KS-E, JG, SM, $M S, G K, M K, I H, M L-T, S K, D H, S K, M V, S G, M D, T G$, $M K, T H$, and ML. DW was supported by the Deutsche Forschungsgemeinschaft (DFG, German Research Foundation, grant number 324392634 - TRR 221).

\section{References}

1.Ljungman P, Hakki M, Boeckh M. Cytomegalovirus in hematopoietic stem cell transplant recipients. Hematol Oncol Clin North Am. 2011;25(1):151-169.

2. Azevedo LS, Pierrotti LC, Abdala E, et al. Cytomegalovirus infection in transplant recipients. Clinics. 2015;70(7):515-523.

3. de la Cámara R. CMV in Hematopoietic stem cell transplantation. Mediterr J Hematol Infect Dis. 2016;8(1):e2016031.

4. Ljungman $\mathrm{P}$, de la Camara R, Cordonnier C, et al. Management of CMV, HHV-6, HHV-7 and Kaposi-sarcoma herpesvirus (HHV-8) infections in patients with hematological malignancies and after SCT. Bone Marrow Transplant. 2008;42(4):227-240.
5. Tomblyn $M$, Chiller $\mathrm{T}$, Einsele $\mathrm{H}$, et al. Guidelines for preventing infectious complications among hematopoietic cell transplant recipients: a global perspective. Preface. Bone Marrow Transplant. 2009;44(8):453455.

6. Emery V, Zuckerman M, Jackson G, et al. Management of cytomegalovirus infection in haemopoietic stem cell transplantation. $\mathrm{Br}$ J Haematol. 2013;162(1):25-39. 
Haematol. 2018;101(1):38-47.

50. Lilleri D, Fornara C, Chiesa A, et al. Human cytomegalovirus-specific $\mathrm{CD} 4+$ and $\mathrm{CD} 8+$ T-cell reconstitution in adult allogeneic hematopoietic stem cell transplant recipients and immune control of viral infection. Haematologica. 2008;93(2):248-256.

51. Ozdemir E, St John LS, Gillespie G, et al. Cytomegalovirus reactivation following allogeneic stem cell transplantation is associated with the presence of dysfunctional anti- gen-specific CD8+ $\mathrm{T}$ cells. Blood. 2002;100(10):3690-3697.

52. Jung J, Lee H-J, Kim S-M, et al. Diagnostic usefulness of dynamic changes of CMV-specific T-cell responses in predicting CMV infections in HCT recipients. J Clin Virol. 2017;87:5-11.

53. Marty FM, Ljungman P, Chemaly RF, et al. Letermovir prophylaxis for cytomegalovirus in hematopoietic-cell transplantation. N Engl J Med. 2017;377 (25):2433-2444.
54. Lindemann M, Eiz-Vesper B, Steckel NK, et al. Adoptive transfer of cellular immunity against cytomegalovirus by virus-specific lymphocytes from a third-party family donor. Bone Marrow Transplant. 2018;53 (10):1351-1355

55. Chemaly RF, Chou $\mathrm{S}$, Einsele $\mathrm{H}$, et al. Definitions of resistant and refractory cytomegalovirus infection and disease in transplant recipients for use in clinical trials. Clin Infect Dis. 2019;68(8):1420-1426. 